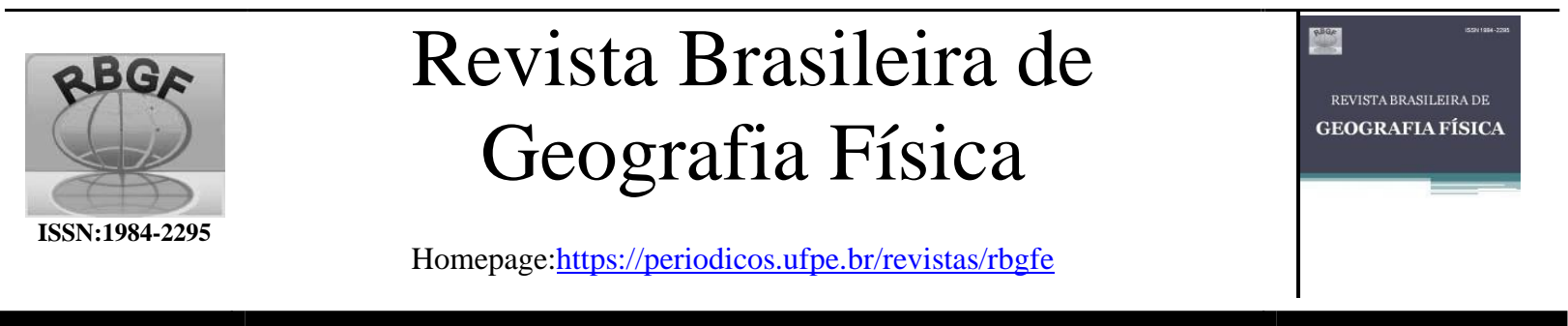

\title{
Avaliação da aplicação de modelos de desprendimento em bacia experimental do semiárido brasileiro
}

\author{
Paulo Roberto de Souza Silveira ${ }^{1}$; George Leite Mamede ${ }^{2}$ \\ 1Doutor em Engenharia Agrícola, UFC, Fortaleza-CE. paulor 1000@ hotmail.com \\ 2Prof. Doutor, UNILAB, Redenção-CE. georgemamede @ hotmail.com \\ Artigo recebido em 12/08/2020 e aceito em 30/11/2020
}

\section{RES U M O}

Granulometria é um dos principais fatores que afetam a dinâmica de sedimentos em bacias hidrográficas, desde seu desprendimento do solo matriz até o transporte ou deposição pela rede de drenagem e reservatório. Objetivou-se com esse trabalho analisar o comportamento granulométrico dos sedimentos em uma bacia do semiárido brasileiro, no bioma Caatinga e analisar a eficiência da aplicação de modelos de estimativa do padrão granulométrico dos sedimentos desprendidos em encostas. O estudo foi realizado na bacia hidrográfica do Pentecoste, situada no semiárido do Ceará. A parte experimental da pesquisa foi realizada na Bacia Experimental do Vale do Curú (BEVC) com coleta de dados pluviométricos e hidrossedimentológicos, amostras de solo da bacia de captação, precipitação diária e de escoamentos superficiais, durante a quadra chuvosa entre os anos de 2015 e 2017. Modelos de desprendimento de sedimentos divulgados na literatura foram testados e aplicados para a área de estudo. Os resultados mostraram que o modelo de desprendimento de sedimento da encosta proposto por Foster et al. (1981) apresentou os melhores resultados quando comparado aos demais, com coeficiente de Nash e Sutcliff (NS) de 0,99, em ambiente de cobertura vegetal mista.

Palavras-chave: Granulometria, Modelo de Foster, Bacia Experimental do Vale do Curú, Bioma Caatinga

\section{Evaluation of the application of detachment models in the experimental basin of the Brazilian semi-arid region}

\begin{abstract}
A B S T R A C T
\section{Introdução}

Dentre os grandes problemas ambientais do planeta, a deterioração dos solos está entre eles, causado, principalmente, pela erosão hídrica. Bertoni e Lombardi Neto (2010) especificam como erosão o processo de retirada e transporte de partículas de solo pelos agentes erosivos: água e/ou vento. Trata-se de um processo natural que consiste na desagregação, transporte e deposição de partículas de solo, no entanto pode ser intensificado por ações antrópicas tornando-se muito prejudicial (Sousa et al. 2020)
\end{abstract}

Size grain is one of the main factors that affect sediment dynamics in watersheds, from its detachment from the soil to the transport or deposition through the drainage network and reservoir. The objective of this research was to analyze the granulometric behavior of the sediments in a Brazilian semi-arid basin in the Caatinga biome and to analyze the efficiency of the application of models for the estimation of the granulometric pattern of sediments detached on slopes. The study was carried out in the Pentecost basin, located in the semi-arid region of Ceará. The experimental part of the research was conducted in the Curú Valley Experimental Basin (BEVC) with rainfall and hydrosedimentological data collection, soil samples from the catchment basin, daily precipitation and surface runoff during the rainy season between 2015 and 2017 . Models of sediment release disclosed in the literature were tested and applied to the study area. The results showed that the slope sediment detachment model proposed by Foster et al. (1981) presented the best results when compared to the others, with Nash and Sutcliff coefficient (NS) of 0.99, under mixed vegetation cover.

Keywords: size grain, Foster model, Experimental Basin of the Curú Valley, Caatinga biome.

Regiões com clima semiárido são propensas ao processo erosivo devido às características do clima, solo e vegetação, e requerem estudos que mostrem quais variáveis são mais determinantes neste processo (Wei et al. 2014; Zhou et al. 2016),

Pesquisas à respeito da dinâmica e erosão do solo focaram principalmente em quantificar o material erodido em um determinado intervalo de tempo. Segundo Lima Neto et al. (2011), muitas pesquisas formularam equações empíricas relacionadas a quantidade de sedimento em uma 
bacia hidrográfica, combinada ou não com outros fatores como forma da bacia, topografia, regime de escoamento, litologia e cobertura vegetal (Walling, 1983; Church \& Slaymaker, 1989; Verstraeten et al., 2003; de Vente \& Poesen, 2005; Minear \& Kondolf, 2009).

Porém, pesquisas têm dirigido atenção às propriedades qualitativas do material erodido, incluindo estabilidade de agregado, mineralogia, matéria orgânica, densidade, forma da partícula e, principalmente, granulometria do sedimento. Tal atenção reside no fato de que, distribuição granulométrica e densidade do sedimento são fatores que determinam se serão transportados ou depositados para determinada condição de fluxo superficial (Stefano \& Ferro, 2002).

O objetivo deste trabalho foi avaliar a aplicação de modelos de desprendimento de solo em uma bacia experimental localizada no semiárido do Estado do Ceará, Brasil.

\section{Material e métodos}

O estudo da dinâmica de sedimentos foi realizado na Bacia Experimental do Vale do Curú (BEVC) a zona rural do município de Pentecoste, pertencente a Universidade Federal do Ceará (Figura 1). No exutório da BEVC está o açude Escola, que apresenta uma capacidade de armazenamento de aproximadamente $51.600 \mathrm{~m}^{3}$. A BEVC compreende uma área de 284,5 ha.

A classificação climática da região segundo Köppen é do tipo Aw - tropical chuvoso sendo influenciada por duas estações no ano, chuvosa no verão-outono e seca no inverno e primavera. As temperaturas médias anuais variam de $22^{\circ} \mathrm{C}$ a $28^{\circ} \mathrm{C}$. Apresenta, em média, umidade relativa do ar em torno de $74 \%$ ao longo do ano e evaporação de $1552,4 \mathrm{~mm}$. A precipitação média anual é de $806 \mathrm{~mm}$ e com alta variabilidade espacial e temporal (Ponce, 1995).

A vegetação predominante é a Caatinga, de caráter hiperxerófilo, termo que, segundo Lemos (2006), é utilizado no Nordeste brasileiro "para designar qualquer comunidade vegetal padronizada pelo seu aspecto fisionômico, resultante da caducifolia de suas espécies no período seco, assumindo caráter tropofítico e refletindo a semiaridez no chamado Polígono das Secas".

De acordo com o mapa base de Reconhecimento dos Solos do Ceará na escala de 1:600.000, que contém as classes de solo para todo o Estado, identificaram-se três diferentes tipos de solo dentro da bacia. Sendo eles Luvissolos e Neossolos.

Os dados pluviométricos na BEVC foram obtidos em uma estação pluviométrica automatizada instalada na área de estudo, que contém um pluviógrafo de Báscula, com aquisição de dados a cada 5 minutos e dois pluviômetros Ville de Paris. Também contará com dados de uma estação meteorológica que funciona na FEVC pertencente a Universidade Federal do Ceará UFC.

Amostras de escoamento foram coletadas em locais específicos da BEVC. A área conta com duas Parcelas do tipo Wischmeyer, que são estruturas que possibilitam a coleta de volume de escoamento superficial o que possibilita a quantificação da perda de água e solo, em escala de lote, com dimensões de $4 \times 22 \mathrm{~m}$. A cobertura vegetal das parcelas estudadas é composta por uma vegetação de Caatinga raleada com 8 anos de pousio na Parcela P1, e, na Parcela P2 uma Caatinga conservada com 40 anos de pousio. A declividade média das parcelas, determinada com auxílio de um nível de mangueira, é de $8,9 \%$ e de 9,7\% para $\mathrm{P} 1$ e $\mathrm{P} 2$, respetivamente.

A parte inferior da parcela foi conectada a um sistema coletor, constituído de 3 tanques com capacidade de 25, 200 e 200 Litros, respectivamente. $\mathrm{O}$ primeiro

tanque é composto de um sistema de 7 janelas. Após o enchimento do primeiro tanque, 1/7 do escoamento superficial da parcela é canalizado para o segundo tanque, que uma vez cheio, o excedente é conduzido para o terceiro tanque. 


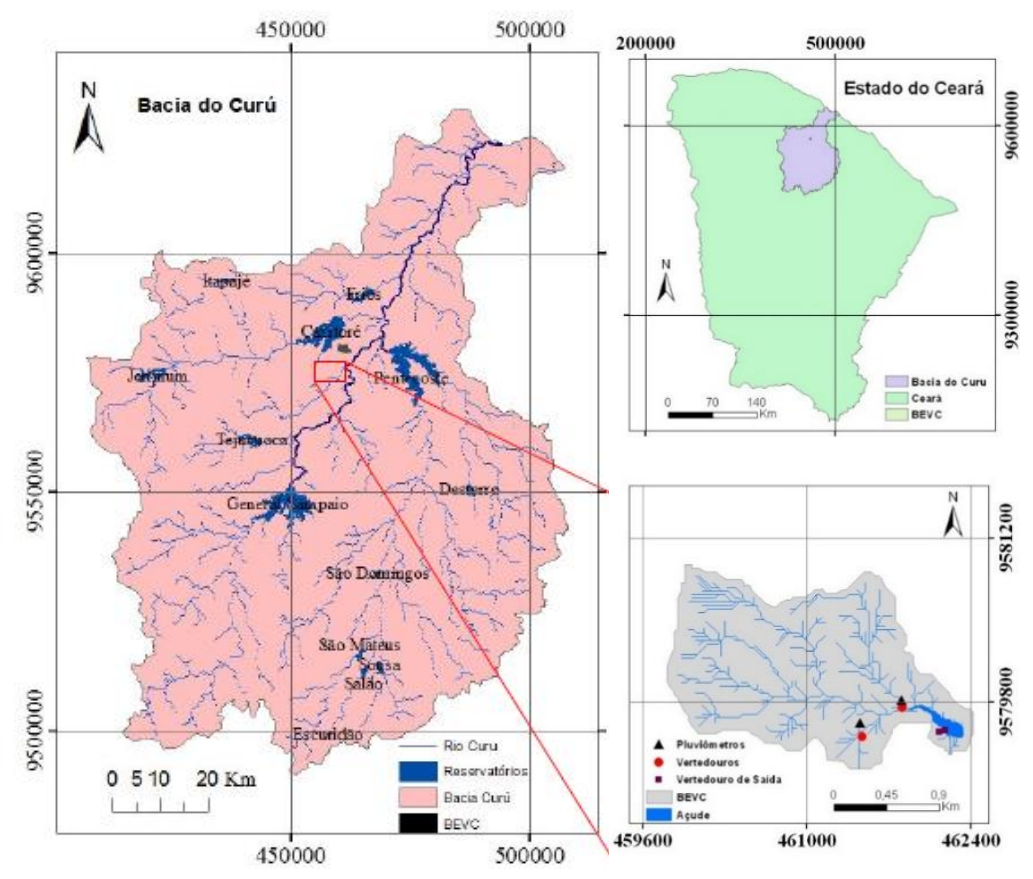

Figura 1. Localização da BEVC.

A análise granulométrica da carga de sedimentos suspensos foi feita através do método de análise do TUBO DE REMOÇÃO PELA BASE ou Bottom. Segundo Carvalho (2008), esse método, comum às análises do material de suspensão e de fundo, objetiva a determinação da granulometria e da concentração do material contido na amostra. $\mathrm{O}$ método é descrito por Carvalho (1994) e objetiva a determinação da granulometria como também da Concentração de Sedimentos Suspensos (CSS) contida na amostra através da precipitação das partículas. O processo é baseado na teoria de Oden (Carvalho, 2008) e na Lei de Stokes (Stokes, 1851)

Na BEVC, definiu-se pontos de coleta de solo e foram realizadas coletas de amostras deformadas, na profundidade de 0 a $20 \mathrm{~cm}$, para análise de granulometria e matéria orgânica. As amostras foram acondicionadas em sacos plásticos e levadas ao Laboratório de Mecânica dos Solos e Pavimentação da Universidade Federal do Ceará (UFC), onde os ensaios foram conduzidos.

As amostras para a análise granulométrica foram preparadas de acordo com a NBR6457, que dispõe sobre o preparo de amostras para compactação e ensaios de caracterização.

Qualificação granulométrica do sedimento desprendido do solo matriz pode ser prevista utilizando modelos empíricos. Nessa pesquisa, 6 modelos foram avaliados: Frere et al. (1975); Young \& Onstad (1976); Young (1980), Foster et al. (1981); Foster et al. (1985) e Carkovic et al. (2015).

Frere et al. (1975) desenvolveram uma relação entre a área superficial específica de cada classe granulométrica e a textura do solo matriz para estimar a distribuição granulométrica do sedimento, ou Padrão Granulométrico de Sedimento Desprendido (PSD), erodido da encosta.

Young e Onstad (1976) desenvolveram equações para prever a composição granulométrica do solo erodido, levando em consideração a distribuição granulométrica do solo matriz, o conteúdo de matéria orgânica e a umidade do solo.

Young (1980) também sugeriu um set de equações para se estimar a granulometria do material desprendido. $\mathrm{O}$ autor destaca que os componentes do solo, argila, silte e areia, são transportados, em sua maior parte, na forma de agregados, principalmente a argila.

Foster et al. (1981) propuseram um método desenvolvido como componente do modelo CREAMS (Chemicals, Runoff e Erosion from Agricultural Management Systems) (USDA, 1980; Knisel, 1980). O método avalia a produção de sedimento sobre várias práticas de manejo da bacia e incorpora princípios fundamentais de erosão, deposição e transporte de sedimentos.

Foster et al. (1985) elaboraram uma série de equações empíricas, baseadas em dados coletados de 28 solos dos EUA, relacionando a textura do solo matriz com a composição granulométrica do sedimento desprendido.

Carkovic et al. (2015) avaliaram as equações empíricas propostas por Foster et al. (1985) para desenvolver novas equações de 
previsão da granulometria do solo desprendido pelos processos erosivos em terrenos não cultivados e afetados por queimadas, equações mais adequadas para as condições do local de estudo.

Através dos modelos foi possível elaborar uma estimativa da distribuição granulométrica dos sedimentos que se desprende do solo pelas forças de cisalhamento, tanto da gota de chuva quando do escoamento superficial.

A análise da aplicação dos modelos propostos foi feita em 3 etapas:

1- Análise da aplicabilidade dos modelos com os dados texturais do solo da parcela P1;

2- Análise da aplicabilidade dos modelos com os dados texturais do solo da parcela P2;

3- Análise da aplicabilidade dos modelos com os dados texturais médios do solo matriz, obtida à partir da análise de 25 amostras coletadas na bacia experimental.

\section{Resultados e discussão}

Caracterização granulométrica dos sedimentos erodidos sob diferentes tipos de coberturas vegetais

Como exposto na metodologia, duas Parcelas Wischmeyer foram instaladas em locais com características vegetacionais distintas de Caatinga, uma raleada (P1) e outra conservada (P2), com a finalidade de se observar, além do escoamento superficial, se a cobertura vegetal poderia influenciar ou não no padrão granulométrico do sedimento desprendido e, consequentemente, erodido.

$\mathrm{O}$ Figura 2 mostra as curvas granulométricas de ambas as parcelas. As curvas são bem parecidas, tanto $\mathrm{P} 1$ como $\mathrm{P} 2$ possuem uma textura arenosa, característica do solo da BEVC.

Analisando a Figura 3, percebe-se que a granulometria dos sedimentos escoados na P1, locada em ambiente de Caatinga raleada, comparada com a P2 locada em ambiente de Caatinga preservada teve característica mais fina, ou seja, maior predominância de argilas e siltes, principalmente quando se observa eventos de cheias próximos.

A possível explicação para tal acontecimento é que a vegetação de maior porte, característica da Caatinga preservada, retém parte da precipitação e diminui a energia cinética da gota quando atinge o solo. Medeiros et al. (2009) relataram que em média $12 \%$ da chuva é interceptada pela cobertura vegetal, variando durante o período da quadra chuvosa de um determinado ano. Quanto menor a energia inerente à gota de chuva menor será sua capacidade de desagregar partículas. Matéria Orgânica e agregados também são fatores que dificultam essa desagregação e impedem que determinado componente do solo se desprenda da estrutura do mesmo e seja passível de erosão.

A textura do solo afeta a estabilidade e a própria formação de agregados e solos com maior teor de argila favorecem a

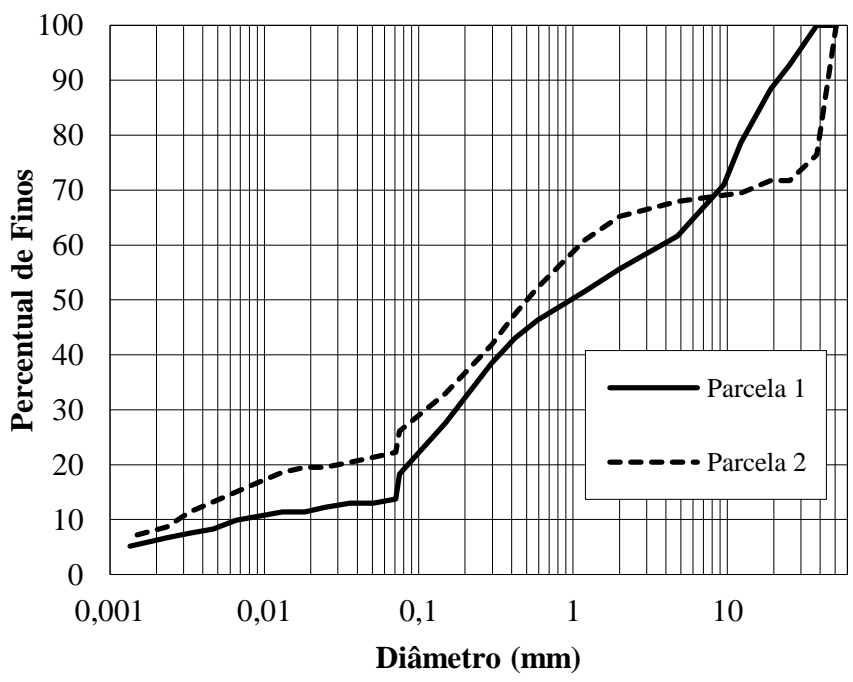

Figura 2: Granulometria de solo das Parcelas Wischmeyer. 


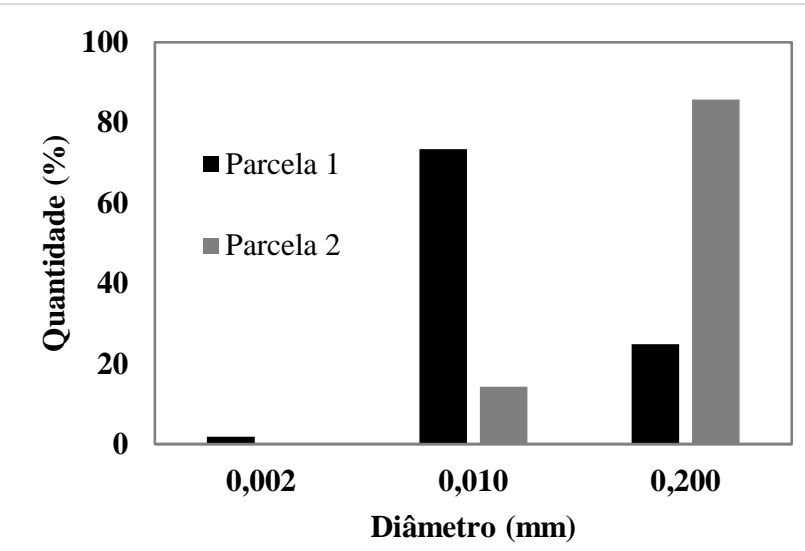

Figura 3: Padrão granulométrico dos sedimentos escoados nas Parcelas Wischmeyer num mesmo período da temporada chuvosa.

agregação. Segundo Vezzani e Mielniczuk (2011), a maior presença de argila contribui para aumentar a aproximação das partículas do solo. De acordo com Santos et al. (2011), horizontes arenosos dificultam a proteção física da matéria orgânica, dificultando a formação de agregados. A BEVC tem uma textura de solo de caráter arenoso, como mostrado na Figura 4, com baixo índice de agregado e matéria orgânica. Lourenço et al. (2017) analisaram as relações entre os teores de Carbono Orgânico no Solo (COS) e os parâmetros umidade do solo e serrapilheira em diferentes condições de Caatinga da BEVC durante quadra chuvosa. Os autores verificaram que o COS da bacia teve um valor médio de $2,24 \%$ e a umidade média foi de $6,61 \%$

$\mathrm{O}$ processo de seletividade de material desprendido de solo foi estudado também por
Farmer (1973), Poesen e Savat (1980) e Issa (2006), os quais demonstraram que há uma preferência de erosão, desprendida, dos materiais mais grosseiros do solo. Podwojewski et al. (2011) relataram diversas alterações no Padrão Granulométrico de Sedimentos Desprendidos (PSD) em virtude de diferenciação na cobertura vegetal. Observaram que uma maior cobertura vegetal no solo superficial contribui para uma retenção de partículas mais finas do solo, atuando como uma espécie de filtro.

A diferenciação no Padrão Granulométrico de Sedimentos Desprendidos (PSD) do solo em locais de diferentes coberturas vegetais é consequência da capacidade de interceptação da precipitação diferenciada para cada cobertura. A presença de

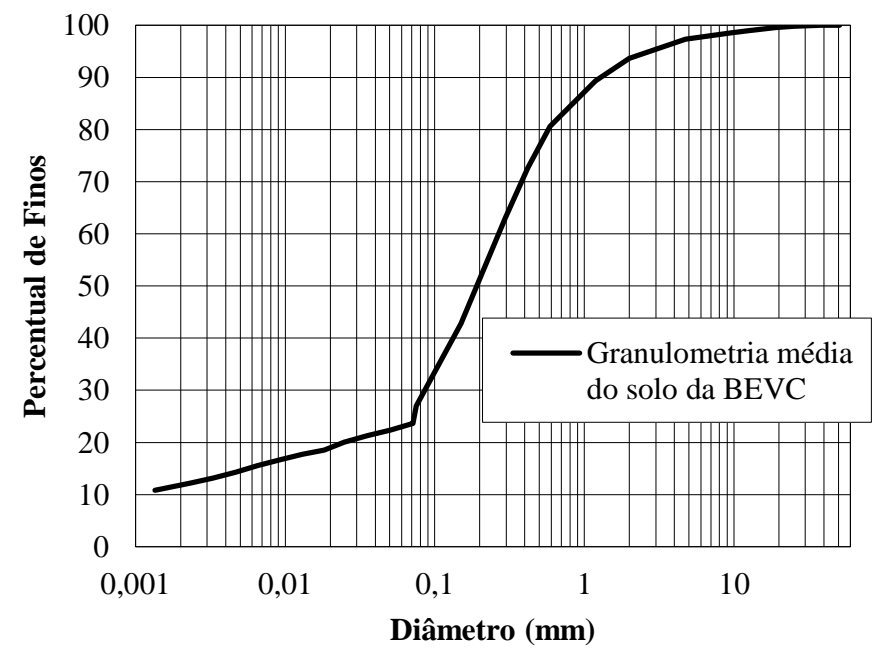

Figura 4: Curva granulométrica média do solo da BEVC. 
vegetação amortece a energia de impacto da gota de chuva no contato com o solo, o que contribui para a redução da desagregação de partículas de sedimentos.

Analisando os resultados granulométricos (Figura 5) para cada evento e parcela, verificou-se que há uma tendência de maior taxa de desagregação do componente do solo silte. Isso pode ser visto claramente na parcela P1 para o evento de 28/01/2016 e de 11/03/2016, as quais tiveram concentrações de silte no solo desprendido de $60,1 \%$ e $73,2 \%$, respectivamente. Os distintos resultados encontrados na parcela $\mathrm{P} 2$ para o evento de 10/03/2016 é provavelmente devido aos baixos valores de escoamento registrados nessa parcela, discutido anteriormente.

Martinez-Mena et al. (1999), analisando a característica granulométrica do sedimento desprendido em ambiente semiárido sob chuva natural e dois tipos de cobertura vegetal, verificaram que a característica do sedimento em todos os eventos e condições de cobertura foi de caráter siltoso

Siltes são partículas facilmente transportáveis e dominam as concentrações no sedimento erodido; tem baixo poder de coesão entre partículas, o que deve beneficiar o seu desprendimento e transporte (Miller \& Baharuddin, 1987; Rienzi et al., 2013; Wang et al., 2014; Nearing et al., 1991).
Segundo Gumiere et al. (2009), nas partículas mais grosseiras, o grau de desprendimento é inversamente proporcional à granulometria da partícula, ou seja, quanto maior à partícula menor a sua possibilidade de se desprender do solo, que está associado ao aumento da massa da partícula. Já para as menores partículas, a possibilidade de desprendimento diminui de acordo com diminuição da partícula, o qual está associado ao aumento do poder de coesão entre as partículas (Farmer, 1973). Ou seja, solos com altos teores de argila e areia tem mais baixas taxas de erodibilidade quando comparados com solos com altos teores de silte.

Analisando a Figura 6, o qual descreve as frequências absolutas dos sedimentos contidos no volume escoado nas parcelas, verificou-se, em todos, um comportamento bimodal. Foster et al. (1985) atentaram para tal acontecimento, afirmando que a distribuição granulométrica de sedimentos erodidos tendiam para um comportamento bimodal em muitos solos, com picos entre 0,02-0,06 $\mathrm{mm}$ e 0,2-1,0 $\mathrm{mm}$. Fato que ocorreu também nos resultados encontrados por Hao et al. (2016), analisando a distribuição granulométrica de sedimentos erodidos em três distintos tipos de solo da China sob precipitação natural, os quais encontraram picos entre as classes 0,002-0,05 mm e 0,5-1,0 mm.

É importante frisar que muitas pesquisas à respeito de Padrão

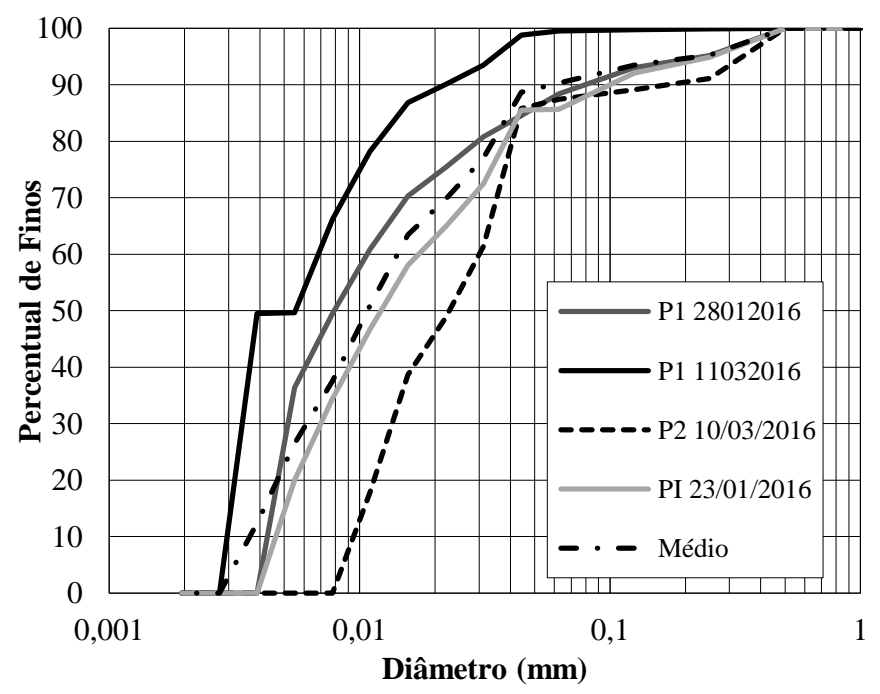

Figura 5: Padrão granulométrico do escoamento superficial das parcelas Wischmeyer em diferentes períodos.

Granulométrico de Sedimentos Desprendidos (PSD) foram feitas em âmbito de laboratório com chuva simulada (Young, 1980; Alberts et al., 1983, Foster et al., 1985, Finkner et al., 1986; Meyer et al., 1992; Farenhorst e Ryan, 1995), como também algumas informações sobre caracterização granulométrica de sedimento erodido sob chuva natural tiveram suas amostras dispersadas para análise, o que pode alterar os resultados, principalmente em solos com altos teores de agregados. 


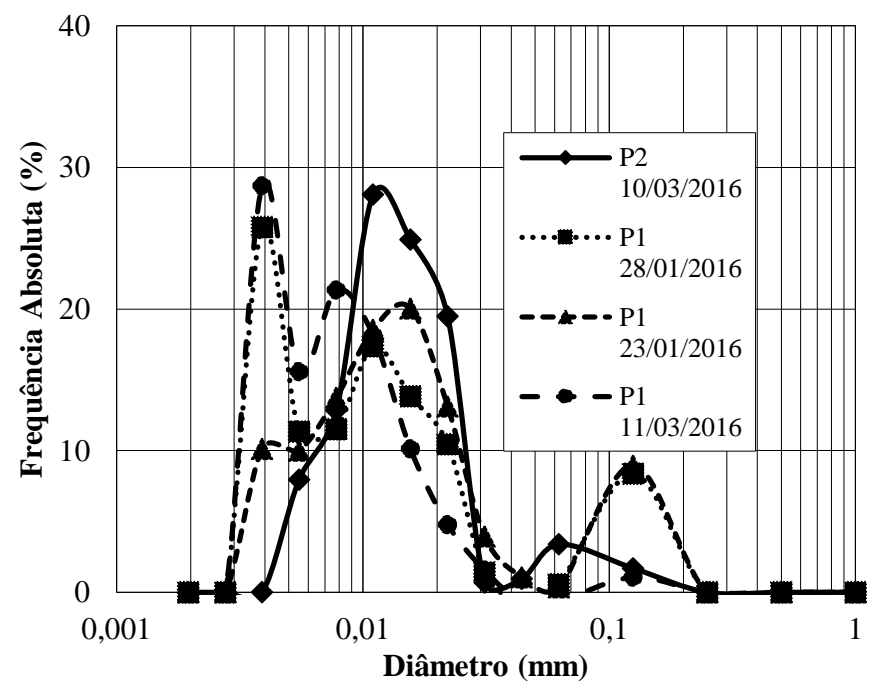

Figura 6: Frequência absoluta das classes dos sedimentos erodidos.

Análise da eficiência dos modelos de desprendimento

\section{Parcela P1}

Para a referida parcela, a análise mostrou que os modelos apresentados na metodologia se mostraram discrepantes comparados uns com os outros, alguns previram com certa acurácia o Padrão de Sedimento Desprendido (PSD) nas amostras escoadas, como os modelos de Young (1980) e Young \& Onstad (1971), com coeficiente de Nash e Sutcliff (NS) de 0,76 e 0,87, respectivamente. Já o modelo proposto por Foster et al. (1985) obteve o menor valor de NS, 0,44. O modelo que previu com mais acurácia o PSD foi o modelo de Foster et al. (1981), com coeficiente de correlação de 0,92 e, consequentemente, menor RMSE, 0,09. A síntese dos resultados pode ser encontrada na Tabela 1.

$\mathrm{Na}$ pesquisa desenvolvida por Carkovic et al. (2015), os resultados foram diferentes quando comparados ao modelo proposto por Foster et al. (1985). No âmbito dessa pesquisa, os resultados corroboraram com os encontrados pelos pesquisadores, atingindo coeficiente NS de 0,71.

De acordo com Young (1980), o desprendimento de partículas do solo depende da textura, densidade e grau de agregação entre as partículas do solo. Foi destacado anteriormente que os sedimentos desprendidos tinham altas concentrações de silte. $\mathrm{O}$ autor destaca que solos com percentual de areia ou argila maiores que $50 \%$, como no caso da BEVC, produzem sedimentos com altas concentrações de partículas de tamanho em torno de $50 \mu \mathrm{m}$, classe do silte. À título de informação, ainda segundo Young (1980), solos argilosos tendem a entregar, no escoamento, sedimento mais grosseiro, isso devido ao alto poder de agregação entre as argilas, o que dificulta o desprendimento dessas partículas. Já solos com teores de silte maiores que $33 \%$ tendem a produzir sedimentos com tamanho entre 20 e $35 \mu \mathrm{m}$.

\section{Parcela P2}

Como na parcela P1, alguns modelos previram com boa acurácia a PSD dos sedimentos contidos no escoamento superficial da parcela P2, com destaque para o modelo de Foster et al. (1985) e Carkovic et al., (2015), que tiveram NS de 0,91 e 0,87 , respectivamente. Ao contrário, o modelo de Young e Onstad (1971), com NS de 0,35. A síntese dos resultados pode ser encontrado na Tabela 2.

Nesse ponto, torna-se importante frisar as discrepâncias entre os modelos quando comparadas as parcelas P1 e P2. O modelo de Foster et al. (1985) apresentou boa acurácia na previsão do PSD da parcela P2 mas em relação a P1 seu comportamento se mostrou contrário com os menores valores de NS. Comportamento parecido com o modelo de Young \& Onstad (1971), apresentando boa acurácia na PSD da parcela P1 mas comportamento contrário na PSD da parcela $\mathrm{P} 2$.

\section{Bacia}

O modelo de Foster et al. (1981) apresentou os melhores resultados ao prever o PSD da BEVC como um todo, obtendo NS de 0,99. Os modelos se comportaram relativamente bem, Carkovic et al. (2015), Young (1980), Young e Onstad (1976) e Frere et al. (1975) obtiveram NS de $0,95,0,90,0,90$ e 0,88 , respectivamente. $\mathrm{O}$ 
modelo que previu com menos acurácia o PSD da bacia foi o de Foster et al. (1985), o qual obteve NS de 0,65. A síntese dos resultados pode ser encontrada na Tabela 3.

Verificou-se nos resultados que os modelos de Foster et al. (1981) e Foster et al. (1985) se comportaram de maneira contrária nas três situações estudadas. A provável explicação para essa discrepância no desempenho dos modelos é que o primeiro tem característica mais empírica, foi construído para ser utilizado em muitas e diferentes situações, tendo como base os preceitos da USLE (Equação Universal de Perda de Solo), sem calibração e coleta de dados para determinação de parâmetros. Já o segundo, foi construído à partir de 28 dados reais de granulometria de solo desprendido em diferentes locais e ambientes dos EUA, ou seja, tem característica mais física.

Martinez-Mena et al. (2002), em estudo realizado do semiárido do mediterrâneo, analisaram as características granulométricas dos sedimentos erodidos sob diferentes intensidades de chuva simulada em dois tipos de solo, constataram que o sedimento erodido tinha sempre característica mais fina que o solo matriz, independente da intensidade e do tipo de solo.

Deizman et al. (1987) conduziram um experimento a fim de avaliar o PSD, usando chuva simulada de $50 \mathrm{~mm} / \mathrm{h}$, em parcelas de declividade entre 8,5 e $9,7 \%$. Os resultados mostraram que a quantidade de chuva, declividade, umidade do solo e textura não dispersada do solo matriz explicavam a variação no PSD. Gabriels e Moldenhauer (1978) conduziram uma série de experimentos em quatro tipos e solo usando chuva simulada de $63,5 \mathrm{~mm} / \mathrm{h}$ durante 90 minutos para avaliar o efeito da textura e intensidade de chuva no PSD. Eles encontraram que o PSD teve altas porcentagens de partículas com diâmetro em torno de $0,05 \mathrm{~mm}$.

Os resultados dessa pesquisa contrariam os encontrados por Meyer et al. (1980 e 1986) os quais observaram que a granulometria do solo desprendido foi similar à do solo matriz, porém com textura levemente mais grosseira do que o solo matriz. A explicação para o fato, segundo os pesquisadores, foi a alta quantidade de agregados no solo erodido; o solo matriz tinha conteúdo de argila superior a $50 \%$.

Tabela 1: Valores de Coeficiente de Nash e Sutcliff (NS) e Raíz do Erro Quadrático Médio (RMSE) gerais dos modelos avaliados para a Parcela 1.

\begin{tabular}{|c|c|c|c|c|c|c|c|}
\hline $\begin{array}{c}\text { Classe } \\
\text { mm }\end{array}$ & Observado & $\begin{array}{c}\text { Foster et al. } \\
1985\end{array}$ & $\begin{array}{c}\text { Carkovic et al. } \\
2015\end{array}$ & $\begin{array}{c}\text { Frere et al. } \\
1975\end{array}$ & Young, 1980 & $\begin{array}{c}\text { Young \& Onstad } \\
1971\end{array}$ & $\begin{array}{c}\text { Foster et } \\
\text { al. } \\
1981\end{array}$ \\
\hline 0,002 & 0,011 & 0,051 & 0,001 & 0,254 & 0,098 & 0,202 & 0,039 \\
\hline 0,004 & 0,304 & 0,120 & 0,153 & 0,269 & 0,214 & 0,322 & 0,239 \\
\hline 0,008 & 0,608 & 0,191 & 0,312 & 0,285 & 0,336 & 0,447 & 0,447 \\
\hline 0,016 & 0,691 & 0,364 & 0,459 & 0,438 & 0,478 & 0,565 & 0,565 \\
\hline 0,031 & 0,776 & 0,538 & 0,606 & 0,591 & 0,620 & 0,684 & 0,684 \\
\hline 0,063 & 0,859 & 0,710 & 0,753 & 0,744 & 0,762 & 0,802 & 0,802 \\
\hline 0,200 & 1,000 & 1,000 & 1,000 & 1,000 & 1,000 & 1,000 & 1,000 \\
\hline NS & - & 0,44 & 0,71 & 0,61 & 0,76 & 0,87 & 0,92 \\
\hline RMSE & - & 0,24 & 0,17 & 0,20 & 0,16 & 0,11 & 0,09 \\
\hline
\end{tabular}

Tabela 2: Valores de Coeficiente de Nash e Sutcliff (NS) e Raíz do Erro Quadrático Médio (RMSE) gerais dos modelos avaliados para a Parcela 2.

\begin{tabular}{c|cccccccc}
\hline Classe & Observado & $\begin{array}{r}\text { Foster et al. } \\
\text { mm }\end{array}$ & & $\mathbf{1 9 8 5}$ & $\mathbf{2 0 1 5}$ & $\mathbf{1 9 7 5}$ & Young, 1980 & $\begin{array}{c}\text { Young \& Onstad } \\
\text { Foster et } \\
\text { al. }\end{array}$ \\
\hline 0,002 & 0,000 & 0,058 & 0,001 & 0,254 & 0,111 & Frere et al. & 0,232 & 0,044 \\
0,004 & 0,070 & 0,179 & 0,175 & 0,370 & 0,287 & 0,393 & 0,278 \\
0,008 & 0,143 & 0,304 & 0,355 & 0,490 & 0,469 & 0,561 & 0,521 \\
0,016 & 0,326 & 0,453 & 0,493 & 0,599 & 0,582 & 0,655 & 0,623 \\
0,031 & 0,510 & 0,602 & 0,631 & 0,708 & 0,696 & 0,749 & 0,726 \\
0,063 & 0,693 & 0,751 & 0,769 & 0,817 & 0,809 & 0,843 & 0,828
\end{tabular}


Revista Brasileira de Geografia Física v.14, n.01 (2021) 396-406.

\begin{tabular}{l|ccccccc}
\multicolumn{1}{c}{0,200} & 1,000 & 1,000 & 1,000 & 1,000 & 1,000 & 1,000 & 1,000 \\
\hline NS & - & 0,91 & 0,87 & 0,49 & 0,65 & 0,35 & 0,57 \\
RMSE & - & 0,10 & 0,12 & 0,24 & 0,20 & 0,27 & 0,22 \\
\hline
\end{tabular}

Tabela 3: Valores de Coeficiente de Nash e Sutcliff (NS) e Raíz do Erro Quadrático Médio (RMSE) gerais dos modelos avaliados para a Bacia.

\begin{tabular}{|c|c|c|c|c|c|c|c|}
\hline $\begin{array}{c}\text { Classe } \\
\mathbf{m m}\end{array}$ & Observado & $\begin{array}{c}\text { Foster et al. } \\
1985 \\
\end{array}$ & $\begin{array}{c}\text { Carkovic et al. } \\
2015 \\
\end{array}$ & $\begin{array}{c}\text { Frere et al. } \\
1975 \\
\end{array}$ & Young, 1980 & $\begin{array}{c}\text { Young \& Onstad } \\
1971 \\
\end{array}$ & $\begin{array}{c}\text { Foster et } \\
\text { al. } \\
1981 \\
\end{array}$ \\
\hline 0,002 & 0,008 & 0,060 & 0,001 & 0,254 & 0,115 & 0,238 & 0,046 \\
\hline 0,004 & 0,245 & 0,108 & 0,181 & 0,326 & 0,220 & 0,338 & 0,279 \\
\hline 0,008 & 0,491 & 0,159 & 0,368 & 0,401 & 0,329 & 0,442 & 0,520 \\
\hline 0,016 & 0,600 & 0,338 & 0,503 & 0,529 & 0,473 & 0,561 & 0,623 \\
\hline 0,031 & 0,709 & 0,519 & 0,639 & 0,657 & 0,617 & 0,681 & 0,726 \\
\hline 0,063 & 0,817 & 0,698 & 0,774 & 0,785 & 0,760 & 0,800 & 0,828 \\
\hline 0,200 & 1,000 & 1,000 & 1,000 & 1,000 & 1,000 & 1,000 & 1,000 \\
\hline NS & - & 0,64 & 0,95 & 0,88 & 0,90 & 0,90 & 0,99 \\
\hline RMSE & - & 0,19 & 0,07 & 0,11 & 0,10 & 0,10 & 0,02 \\
\hline
\end{tabular}

\section{Conclusões}

Os resultados mostraram que o modelo de desprendimento de sedimento da encosta proposto por Foster et al. (1981) apresentou os melhores resultados quando comparado aos demais, com coeficiente de Nash e Sutcliff (NS) de 0,92, em ambiente de Caatinga raleada. Em ambiente de Caatinga preservada, o modelo proposto por Foster et al. (1985) apresentou os melhores resultados, com coeficiente NS de 0,91. Em ambiente misto, o modelo proposto por Foster et al. (1981) apresentou os melhores resultados, com coeficiente NS de 0,99 .

\section{Referências}

Alberts, E.E., Wendt, R.C., Piest, R.F.

1983. Physical and chemical properties of eroded soil aggregates. Transations of the American Society of Agricultural Engineers 26, 465-471.

Bertoni, J., Lombardi Neto, F, 1999. Conservação do solo. São Paulo - SP, Ícone.

Carkovic, A.B., Pastén, P.A., Bonilla, C.A, 2015. Sediment composition for the assessment of water erosion and nonpoint source pollution in natural and fire-affected landscapes. Sci. Total Environ 512, 26-35.

Carvalho, N.O, 1994. Hidrossedimentologia Prática. CPRM e ELETROBRÁS. Rio de Janeiro, RJ.

Carvalho, N.O, 2008. Hidrossedimentologia Prática. $2^{\mathrm{a}}$ ed, Interciência. Rio de Janeiro, RJ.
Church, M., Slaymaker, O, 1989. Desequilibrium of Holocente sediment yield in glaciated. British Columbia, Nature 337, 452-454.

Diezman, M.M., Mosttaghimi, S., Shanholtz, V.O., Mitchell, J. K, 1987. Size distribution of eroded sediment from two tillage systems. Transations of the ASAE 30, 1642-1647.

Farmer, E.E, 1973. Relative detachability of soil particles by simulated rainfall. Soil Sci. Soc. Am. J., 37, 629-633.

Foster, G.R., Lane, L.J.; Nowlin, J.D.; Laflen, J.M., Young, R.A, 1981. Estimating erosion and sediment yield on field sized areas. Transactions of the Asae 24, 1253-1262.

Foster, G.R., Young, R.A., Neibling, W.H, 1985. Sediment composition for nonpoint source pollution analyses. Trans. Asae 28, 133-139.

Frere, M.H., Onstad, C.A., Holtan, H.N, 1975. ACTMO, an agricultural chemical transport model. U.S. Agricultural Research Service. ARS-H-3, 1-11.

Gumiere, S.J., Le Bissonnais, Y., Raclot, D, 2009. Soil resistance to interrill erosion: Model parameterization and sensitivity. Catena 77, 274-284.

Gabriels, D.; Moldenhauer, W.C, 1978. Size distribution of eroded material from simulated rainfall: Effect over a range of texture. Soil Science Society of America Journal 42, 954958.

Hao, Y., Yang, Y., Liu, B., Liu, Y., Gao, X., Guo, Q, 2016. Size characteristics of sediment eroded from three soils in China under natural rainfall. Journal of Soils and Sediments 16, 2153-2165. 
Issa, O. M., Le Bissonnais, Y., Planchon, O., FavisMortlock, D., Silvera, N., Wainwright, J, 2006. Soil detachment and transport on field and laboratory-scale interrill areas: erosion processes and the size selevity of eroded sediment 31, 929-939.

Hudson, N, 1971. Soil Conservation. Cornell University Press, Ithaca, New York.

Lemos, J. R, 2006. Florística, estrutura e mapeamento da vegetação de caatinga da estação ecológica de Aiuaba, Ceará. Tese de doutorado. Universidade de São Paulo.

Lourenço, V.R., Pereira, F.J.S., Teixeira, D.B.S., Teixeira, L.M.N., Costa, C.A.G, 2017. Influência relativa dos parâmetros umidade e serapilheira no teor de carbono em diferentes manejos da Caatinga. III Simpósio Brasileiro de Recursos Naturais do Semi-Árido - SBRNS, Fortaleza-Ceará, Brasil.

Martinez-Mena, M., Rogel, J.A., Albaladejo, J., Castillo, V.M, 2002. Relations between interrill erosion processes and sediment particle size distribuition in a semiarid Mediterranean area of SE of Spain. Geomorphology 45, 261-275.

Medeiros, P.H.A., Araújo, J.C., Bronstert, A, 2009. Interception measurements and assessment of Gash model performance for a tropical semiarid region. Revista Ciência Agronômica 40, 165-174.

Meyer, L.D., Line, D.E., Harmon, W.C, 1992. Size characteristics of sediment from agricultural soils. Journal of Soil and Water Conservation 47, 107-111.

Meyer, L.D, 1981. How rain intensity affects interrill erosion. Transactions of the American Society of Agricultural Engineers 23, 14721475.

Meyer, L.D., Harmon, W.C, 1984. Susceptibility of agricultural soils to interrill erosion. Soil Science Society of America Journal 48, 11521157.

Meyer, L.D., Harmon, W.C., McDowell, L.L, 1980. Sediment sizes eroded from crop row sideslope. Transactions of the American Society of Agricultural Engineers 23, 891-898.

Miller, W.P., Baharuddin, M.K, 1987 Particle size of inter-rill-eroded sediments from highly weathered soils, Soil Sci. Soc. Am. J. 51, 16101615.

Minear, J.T., Kondolf, G.M, 2009. Estimating reservoir sedimentation rates at large spatial and temporal scales: A case study of California, Water Resources Research 45.
Nearing, M., Foster, G.R., Lane, L.J., Finkner, S.C, 1989. A process-based soil erosion model for USDA-Water Erosion Prediction Project technology. Trans. ASAE 32, 1587-1593.

Nearing, M.A., Bradford, J.M., Parker, S.C, 1991. Soil detachment by shallow flow at low slopes. Soil Sci. Soc. Am. J. 55, 339-344.

Poesen, J., Savat, J, 1980. Particle-size separation during erosion by splash and runoff', in de Boodt, M. and Gabriels, D. (Eds)

Podwojewski, P., Janeau, J.L., Grellier, S., Valentin, C., Lorentz, S., Chaplot, V, 2011. Influence of grass soil cover on water runoff and detachment under rainfall simulation in a subhumid South African degraded rangeland. Earth Surface Processes and Landforms 36, 911-922.

Ponce, V.M, 1995. Management of droughts and floods in the semiarid Brazilian northeast. The case for conservation. Journal of Soil and Water Conservation 50, 422-431.

Rienzi, E.A., Fox, J.F., Grove, J.H., Matocha, C.J. Interrill erosion in soils with different land uses: The kinetic energy wetting effect on temporal particle size distribution. Catena 107, 130-138.

Santos, D.C., Pillon, C.N., Flores, C.A., Lima, C.L.R., Cardoso, E.M.C., Pereira, B.F., Magrich, A.S, 2011. Agregação e frações físicas da matéria orgânica de um Argissolo Vermelho sob sistemas de uso no bioma Pampa. Revista Brasileira de Ciência do Solo 35, 1735-1744.

Sousa, M, M, M, et al. Características pluviométricas determinantes no transporte de sedimentos em microbacia com vegetação raleada no semiárido tropical. Revista Caatinga 33, 785-793.

Stefano, C., Ferro, V, 2002. Linking clay enrichment and sediment delivery processes. Biosystems Engineering 81, 465-479.

Stokes, G.G, 1851. On the effect of the internal friction on the motion of pendulums, Cambridge Philos. Trans., Part 2 2, 8- 106.

U.S. 1980. Department of Agriculture (USDA). CREAMS - A Field Scale Model for Chemicals, Runoff, and Erosion from Agricultural Management Systems. Conservation Research Report No. 26. USDA, Sci. and Educ. Admin.

de Vente, J., Poesen, J, 2005. Predicting soil erosion and sediment yield at the basin scale: Scale issues and semi-quantitative models, Earth-Science Reviews 71, 95-125.

Verstraeten, G., Poesen, J., de Vente, J., Koninckx, $\mathrm{X}, 2003$. Sediment yield variability in Spain: a quantitative and semiquanlitative analysis using reservoir sedimentation rates. Geomorphology 50, 327-348. 
Vezzani, F. M., Mielniczuk, J, 2011. Agregação e estoque de carbono em Argissolo submetido a diferentes práticas de manejo agrícola. Revista Brasileira de Ciência do Solo 5, 213-223.

Young, R.A, 1972. The role of rainfall impact and surface flow in soil detachment and transport. Unpublished Ph.D. Thesis, South Dakota State University.

Young, R.A., Wiersma, J.L, 1973. Role of rainfall impact in soil detachment and transport. Water Resources Research 9, 1629-1636.

Young, R.A., Onstad, C.A, 1978. Characterization of rill and interrill eroded soil. Transactions of the American Society of Agricultural Engineers 21, 1126-1130.

Young, R.A., Wiersma, J.L, 1973. Role of rainfall impact in soil detachment and transport. Water Resources Research 9, 1629-1636.
Walling, D.E, 1983. The sediment delivery problem. Journal of Hydrology 65, 209-237.

Wang, L., Shi, Z.H., Wang, J., Fang, N.F., Wu, G.L., Zhang, H.Y, 2014. Rainfall kinetic energy controlling erosion processes and sediment sorting on steep hillslopes: A case study of clay loam soil from the Loess Plateau, China. Journal of Hydrology 512, 168-176.

Warrington, D.N., Mamedov, A.I., Bhardwaj, A.K., Levy, G.J, 2009. Primary particle size distribution of eroded material affected by degree of aggregate slaking and seal development. European Journal of Soil Science 60, 84-93.

Wei, W. et al., 2014. Effects of surficial condition and rainfall intensity on runoff in a loess hilly area, China. Journal of hydrology 513, 115-126. 